\title{
Consistencia interna de la versión en español del cuestionario medida de calidad de vida de la diabetes, en Cúcuta (Colombia)
}

\section{Internal consistency of the Spanish version of the questionnaire measure of quality of life of diabetes, in Cucuta (Colombia)}

\section{Consistência interna da versão em espanhol do questionário medida de qualidade de vida da diabetes, em Cúcuta (Colômbia)}

\author{
Luz Marina Bautista-Rodríguez ${ }^{1}$, Gloria Esperanza Zambrano-Plata ${ }^{2}$
}

Forma de citar: L. M. Bautista, G. E. Zambrano, "Consistencia interna de la versión en español del cuestionario medida de calidad de vida de la diabetes, en Cúcuta (Colombia)", Respuestas, vol. 20, no. 1, pp. 51- 66, 2015.

Recibido:

7 de Mayo 2014

Aceptado:

26 de Septiembre 2014

\footnotetext{
'Magister en Enfermería con Énfasis en Gerencia luzmarinabr@ufps.edu.co Universidad Francisco de Paula Santander Cúcuta- Colombia

${ }^{2}$ Doctora en Educación Universidad Francisco de Paula Santander Cúcuta- Colombia
}

\section{Resumen}

Antecedentes: En Colombia desde 1997 se implementa la Encuesta de Calidad de Vida con el objetivo de obtener información básica para la medición de las condiciones de vida de los colombianos y la obtención de indicadores de pobreza y desigualdad [1]. El impacto de las enfermedades crónicas y sus tratamientos tradicionales se han evaluado en términos de morbilidad y mortalidad, sin embargo se ha despertado el interés por la evaluación de la calidad de vida de las personas, debido a que esta es una importante variable de resultado de las intervenciones [2]. Objetivo: Determinar la confiabilidad de la versión en español del cuestionario medida de calidad de vida específica para Diabetes; Métodos: Se realizó estudio cuantitativo descriptivo metodológico, la muestra estuvo constituida por 287 pacientes que asisten a consulta o control de seguimiento por médico o enfermería a las Unidades Básicas de la Empresa Social del Estado Instituto Municipal de Salud, el instrumento utilizado es el cuestionario medida de calidad de vida específica para Diabetes versión español. Resultados: El coeficientes de consistencia interna de los factores del instrumento, oscilo entre 0,29 a 0,88 , se evidencia una baja confiabilidad para la subescala preocupación por aspectos futuros de la enfermedad $(\alpha=0,29)$ y una confiabilidad aceptable para las subescalas de satisfacción con el tratamiento, impacto del tratamiento y preocupación por aspectos sociales y vocacionales. Conclusión: Este estudio demostró que el cuestionario medida de calidad de vida específica para Diabetes (DQOL) en su versión en español tiene una confiabilidad elevada $(\alpha=0,88)$ y una estructura factorial similar a la encontrada en estudios realizados en otras poblaciones.

Palabras claves: Calidad de Vida, Confiabilidad, Cuestionario, Diabetes.

\footnotetext{
Abstract

Background: In Colombia since 1997 Quality of Life Survey in order to obtain basic information for measuring the lives of Colombians and obtaining indicators of poverty and inequality is implemented. [1] The impact of chronic diseases and their
} 
traditional treatments have been evaluated in terms of morbidity and mortality, however, has aroused interest in the evaluation of the quality of life of people, because this is an important outcome variable interventions. [2] Objective: To determine the reliability of the Spanish version of the questionnaire measuring specific quality of life for Diabetes. Methods: Methodological quantitative descriptive study was conducted, the sample consisted of 287 patients attending consultation or control to the Basic Units of the State Social Enterprise Municipal Institute of Health, the instrument used is the questionnaire measuring specific quality of life for Diabetes version Spanish. Results: The internal consistency coefficients of the factors of the instrument ranged from 0.29 to 0.88 , low reliability for the subscale concerns about future aspects of the disease $(\alpha=0.29)$ and acceptable reliability for the subscales evidenced satisfaction with treatment, impact of treatment and concern for social and vocational aspects. Conclusion: The questionnaire measuring specific quality of life for Diabetes in the version in Spanish, is reliable for use in diabetic patients in Colombia.

Keywords: Quality of Life, Reliability, Questionnaire, Diabetes.

\section{Resumo}

Antecedentes: Na Colômbia desde 1997 se programou a pesquisa de Qualidade de Vida com o objetivo de obter informação básica para a medição das condições de vida dos colombianos e a obtenção de indicadores de pobreza e desigualdade [1]. O impacto das doenças crónicas e seus tratamentos tradicionais se têm avaliado em termos de morbidez e mortalidade, não entanto, se tem despertado o interesse pela avaliação da qualidade de vida das pessoas, devido a que esta é uma importante variável de resultado das intervenções [2]. Objetivo: Determinar a confiabilidade da versão em espanhol do questionário medida de qualidade de vida específica para Diabetes; Métodos: Realizouse estudo quantitativo descritivo metodológico, a amostra esteve constituída por 287 pacientes que assistem a consulta ou controle de seguimento por médico ou enfermagem às Unidades Básicas da Empresa Social do Estado - Instituto Municipal de Saúde, o instrumento utilizado é o questionário medida de qualidade de vida específica para Diabetes versão espanhol. Resultados: O coeficiente de consistência interna dos fatores do instrumento oscilou entre 0,29 a 0,88 , se evidenciou uma baixa confiabilidade para a subescala preocupação por aspectos futuros da doença $(\alpha=0,29)$ e uma confiabilidade aceitável para as subescalas de satisfação com o tratamento, impacto do tratamento e preocupação por aspectos sociais e vocacionais. Conclusão: Este estudo demostrou que o questionário medida de qualidade de vida específica para Diabetes (DQOL) em sua versão em espanhol tem uma confiabilidade elevada $(\alpha=0,88)$ e uma estrutura fatorial similar à encontrada em estudos realizados em outras populações.

Palavras-chaves: Qualidade de Vida, Confiabilidade, Questionário, Diabetes.

\section{Introducción}

A lo largo del tiempo distintos autores y organizaciones han intentado conceptualizar la calidad de vida desde distintos enfoques, el constructo ha evolucionado desde una concepción básicamente materialista, en la que primaban los aspectos objetivos de nivel de vida, pasando por la perspectiva donde los aspectos subjetivos se constituyen en el sustento fundamental, hasta la situación actual, en la que se adjudica la subjetividad y el carácter multidimensional del constructo, de forma que, aunque no hay un consenso único, parece que hay un cierto acuerdo en aceptar valorarla de forma multidimensional, 
identificando componentes objetivos y subjetivos [3].

La Calidad de vida es un concepto complejo que refleja aspectos sociales e individuales, objetivos y subjetivos y múltiples ámbitos como son el físico, emocional, educativo o laboral. Desde diversas disciplinas se ha abordado también el concepto de Calidad de Vida. Así, en Medicina tuvo un importante significado en el siglo pasado debido al incremento de enfermedades crónicas y a la posibilidad del diagnóstico temprano de las mismas y su posterior tratamiento, lo que suponía prolongar la vida de los pacientes que antes tendrían dificultades para sobrevivir. Comenzó a planearse la idea de no sólo la cantidad, sino la calidad de los años ganados a partir de las anteriores intervenciones [4].

El constructo Calidad de vida según la revisión de literatura está conformado por dos dimensiones: la dimensión objetiva (condiciones de vida experimentadas por las personas) y la dimensión subjetiva (la valoración del individuo sobre distintos aspectos de su existencia. Así mismo en [5] han reflejado con claridad el constructo Calidad de Vida y la entienden constituida por tres elementos en interacción: 1.- Las condiciones objetivas de vida, o descripción objetiva de los individuos y sus circunstancias. 2.- El bienestar subjetivo, definido como la satisfacción personal con esas condiciones y con el estilo de vida. 3.- Los valores personales $\mathrm{y}$ aspiraciones

En [6], define la calidad de vida como "la percepción del individuo de su posición en la vida en el contexto de la cultura y sistema de valores en los que vive y en relación con sus objetivos, expectativas, estándares y preocupaciones". Se trata de un concepto muy amplio que está influido de modo complejo por la salud física del sujeto, su estado psicológico, su nivel de independencia, sus relaciones sociales, así como su relación con los elementos esenciales de su entorno. De este modo, la calidad de vida es un constructo multidimensional que comprendería tres dimensiones fundamentalmente:

- Dimensión física: percepción del estado físico o la salud, entendida como ausencia de enfermedad, los síntomas producidos por la enfermedad, y los efectos adversos del tratamiento.

- Dimensión psicológica: percepción del individuo de su estado cognitivo y afectivo como el miedo, la ansiedad, la incomunicación, la pérdida de autoestima, la incertidumbre del futuro, etc. También incluye las creencias personales, espirituales y religiosas como el significado de la vida y la actitud ante el sufrimiento.

- Dimensión social: percepción del individuo de las relaciones interpersonales y los roles sociales en la vida como la necesidad de apoyo familiar y social, la relación médicopaciente y el desempeño laboral.

Se han establecido aspectos claves para la medición de la Calidad de Vida, "se reconoce que el significado de las experiencias valoradas positivamente varía a lo largo del tiempo y entre distintas culturas. En la medición se reconoce la importancia de reflejar estas experiencias individuales y del grupo cultural". La medida cuantitativa de calidad de vida representa la posición en un continuo que va de lo mejor a lo peor. De igual manera, la medida se centra en aspectos clave de la existencia que pueden ser mejorados como: a) el grado en que están satisfechas las necesidades básicas; b) el grado en que se han alcanzado logros sociales y materiales; c) la posibilidad de elecciones y oportunidades, y de actuar con base a ellas y d) el grado en que los ambientes permiten a las personas mejorar. La medida utiliza una gran variedad de dominios de vida que están ampliamente aceptados como indicadores claves de plenitud e interconexión de la vida [4]. 
No. 1

Enero - Junio 2015

ISSN 0122-820X

PP: 51-66
El principal objetivo de una escala es determinar el valor de una variable de forma tan precisa como sea posible. Con ello se muestra su utilidad y, por ende, su calidad [7]. Los instrumentos de medida que valoran ciertos constructos tienen que demostrar que poseen las propiedades psicométricas de validez y fiabilidad, esto indica si un instrumento mide de forma adecuada las variables que se pretenden evaluar con facilidad y eficiencia [8], [9]. Sus propiedades deben calcularse con cada una de las muestras en las que se aplica pues no es una propiedad inherente del instrumento y podría ser fiable y válido con una muestra de participantes pero no serlo con otra muestra [8]. La validez, en términos generales, "se refiere al grado en que un instrumento realmente mide la variable que pretende medir" [9] y la Confiabilidad, "hace referencia a la consistencia de una medición, si la escala o el test funciona de manera similar bajo diferentes condiciones, dependientes del mismo instrumento, del tiempo de aplicación, del que hace la medición, de los sujetos, de la interacción entre estas fuentes y del error aleatorio puro" [10].

Un instrumento de medida es fiable si proporciona aproximadamente el mismo tipo de respuestas para diferentes grupos de sujetos o en repetidas aplicaciones a un mismo grupo de sujetos. Además, será válido si correlaciona con las puntuaciones de otros instrumentos que miden constructos donde es esperable una convergencia. La fiabilidad de la consistencia interna del instrumento se puede estimar con el alfa de Cronbach [7], [8], [11]. El método de consistencia interna basado en el coeficiente de fiabilidad alfa de Cronbach permite estimar la fiabilidad de un instrumento de medida a través de un conjunto de ítems que se espera que midan el mismo constructo o una única dimensión teórica de un constructo latente. La medida de la fiabilidad mediante el alfa de Cronbach asume que los ítems (medidos en escala tipo Likert o también es posible para ítems dicotómicos) miden un mismo constructo y que están altamente correlacionados entre sí. Al menos se necesitan dos ítems para poder estimar el valor del coeficiente alfa de Cronbach y cuanto mayor el número de ítems mayor será la fiabilidad de la escala [8].

En lo relacionado a la forma de medir la calidad de vida, se tuvo en cuenta lo multifactorial del concepto, que generalmente representa un constructo multidimensional y dinámico [12], se realizó el análisis factorial asumiendo las cinco subescalas establecidas por los autores del instrumento DQOL. El análisis factorial es una técnica estadística multivariante cuyo principal propósito es sintetizar las interrelaciones observadas entre un conjunto de variables en una forma concisa y segura como una ayuda a la construcción de nuevos conceptos y teorías. El Análisis Factorial puede ser exploratorio o confirmatorio, el análisis exploratorio se caracteriza porque no se conocen a priori el número de factores y es en la aplicación empírica donde se determina este número. Por el contrario, en el análisis de tipo confirmatorio los factores están fijados a priori, utilizándose contrastes de hipótesis para su corroboración [13], [14].

Con los factores rotados, cada una de las variables tendrá una correlación cercana a 1 con uno de los factores y cercana a 0 con el resto de los factores. Hay dos sistemas básicos de rotación de factores: 1) ortogonal, que mantiene la independencia entre los factores rotados, en este método se incluyen el varimax, quartimax y equimax; y 2) no ortogonal, que proporciona nuevos factores rotados que guardan relación entre sí, dentro de este grupo están el oblimin, promax y orthoblique [13]. El método Varimax, es el más usado, se obtiene mediante una matriz de componentes rotados que indica la correlación existente entre cada una de las variables y su correspondiente factor, es lo que se denomina saturaciones, que toma valores entre $-1 \mathrm{y}+1$ [13], [15].

Con la rotación Varimax se busca maximizar las ponderaciones a nivel del factor; es decir, se espera que cada ítem o variable 
sea representativo en solo uno de ellos, con el fin de minimizar al máximo el número de variables dentro de cada factor. Para hacer esta evaluación desde el punto de vista estadístico se recurre a la significancia de las ponderaciones, así: valores menores a $|0,3|$ se consideran no significativos; entre 0,3 y 0,5 de aporte mínimo; entre 0,5 y 0,7 de aporte significativo, y valores mayores a 0,7 son consideradas relevantes $\mathrm{y}$, generalmente, son el objetivo del análisis. Sin embargo, es frecuente que en ocasiones valores mayores a 0,3 también sean considerados para los análisis [16].

La medida objetiva emplea instrumentos cuantitativos que informan sobre frecuencias y cantidades de indicadores observables. La medida subjetiva utiliza la expresión de grados de satisfacción sobre aspectos de la vida u otro tipo de evaluaciones subjetivas, incluyendo componentes cognitivos y afectivos [4].

La calidad de vida ha sido motivo de interés creciente para los clínicos en las tres últimas décadas debido a que se le "considera una variable de desenlace muy importante de la atención médica, y para medirla se han construido instrumentos genéricos y específicos que varían de acuerdo con el tipo de pacientes en estudio, escenario de administración y tipo de cuestionario (formato breve, autoadministración, entrevista, por teléfono o por correo)" [17]. Los instrumentos genéricos se utilizan para evaluar un amplio rango de características aplicables a diversas enfermedades o condiciones; en cambio, los instrumentos específicos tienen un enfoque dirigido a las características más relevantes de la enfermedad o condición en estudio, así como de los pacientes.

Las escalas genéricas que miden la calidad de vida "permiten valorar grupos y poblaciones en general, en cambio las escalas específicas permiten analizar un fenómeno, problema o enfermedad u otras variables relacionadas con la calidad de vida" [17]. La utilización de diferentes escalas y cuestionarios para medir la variable calidad de vida, "puede usarse para planear programas de tratamiento a corto plazo y para evaluar a grupos con diagnósticos específicos". [18]

Entre los instrumentos que valoran la calidad de forma específica está elDQOL(Medida de la Calidad de Vida de la Diabetes), Cuestionario que ha sido validado en países como España y México, diseñado inicialmente por Investigadores de National Institute of Health del grupo de investigación Ensayo de Control y Complicaciones de la Diabetes (DCCT) en 1988, la versión utilizada en la investigación corresponde a la original traducida al inglés [19]. El DQOL mide la calidad de vida de personas con diabetes, está compuesto por 43 ítems que forman 4 dimensiones: Satisfacción con el tratamiento (15 ítems), Impacto del tratamiento (17 ítems), Preocupación social/ vocacional (7 ítems) y Preocupación relativa a los efectos futuros de la diabetes (4 ítems). Cada ítem tiene 5 opciones de respuesta de tipo Likert que puntúan del 1 al 5 . En la subescala de satisfacción las respuestas a cada ítem oscilan entre muy satisfecho (1 punto) a nada satisfecho (5 puntos). En las otras tres subescalas el rango de las respuestas es el siguiente: nunca (1 punto) a siempre (5 puntos). Puede obtenerse una puntuación total y una puntuación por subescalas. Además, hay que tener en cuenta que una menor puntuación implica una mejor calidad de vida" [6].

Los datos de fiabilidad y validez del cuestionario referido por sus autores originales son adecuados, encontrándose que el DQOL y sus cuatro escalas tenían un alto grado de consistencia interna ( $r$ de Cronbach $=0,66-0,92)$ y una excelente fiabilidad testretest $(r=0,78-0,92)[20]$. En otro estudio [6] se encontró al igual que los autores de la escala original, una adecuada consistencia interna tanto en las subescalas como en el total del DQOL (DQOL-Satisfacción con el tratamiento $=0.68$; DQOL-Impacto del tratamiento $=0.87 ; \quad$ DQOL-Preocupación
Enero - Junio 2015 ISSN 0122-820X PP: 51-66 
No. 1

Enero - Junio 2015

ISSN 0122-820X

PP: 51-66 por aspectos sociales-vocacionales $=0.70$; DQOL-Preocupación por efectos futuros diabetes $=0.59$; DQOL-Total, $=0.89$ ).

El DQOL "se caracteriza por adecuada consistenciainterna, con alphas de Cronbach de 0.47 a 0.92 , y altos coeficientes de estabilidad temporal, que van desde 0.78 a 0.92 . En lo que respecta a la validez de constructo, se ha probado que el DQOL se relaciona de manera moderada y consistente con medidas de síntomas psicológicos, bienestar y ajuste a enfermedad, y con medidas genéricas de calidad de vida. Además se cuenta con evidencia de validez discriminada del DQOL. Las puntuaciones de esta escala se relacionan significativamente con la severidad y número de complicaciones médicas, y con el tipo de tratamiento médico de los pacientes" [6].

La versión en castellano del DQOL muestra índices de consistencia interna adecuados y congruentes con los reportados para la versión original en inglés (en rangos de 0.46 a 0.87) [20]. Además, la estructura factorial de la versión en castellano del DQOL fue la teóricamente esperada según lo encontrado en otros estudios [6], [19], [21]. El total de las preguntas se agruparon en los cuatro factores que corresponden a las dimensiones de calidad de vida que evalúa el instrumento de acuerdo a su diseño original, Finalmente, se encontraron relaciones positivas, moderadas y estadísticamente significativas entre prácticamente todas las subescalas y total del DQOL lo que da prueba de la validez convergente del instrumento. La forma de calificación del DQOL que se utilizó para este análisis de correlación fue la sugerida por los autores originales, en donde un incremento en la puntuación significa un mayor deterioro en la calidad de vida [19].

\section{Materiales y métodos}

Serealizóun estudiodescriptivo metodológico. La población estaba conformada por 287 pacientes adultos con diagnóstico de diabetes mellitus tipo 2, usuarios de los programas de crónicas de las Unidades Básicas Libertad, Comuneros o Puente Barco Leones de la ESE IMSALUD. Con un nivel de confianza del $97 \%$, un margen de error de $3 \%$ y una probabilidad de $50 \%$, se calculó una muestra de 287 pacientes. Los pacientes se seleccionaron mediante un muestreo probabilístico aleatorio simple, para esto se realizó un listado general de pacientes que asistían a consulta o control en las Unidades Básicas seleccionadas.

Para la recolección de información se utilizó el cuestionario DQOL, por ser un instrumento breve y sencillo que puede ser auto administrado, que permite su aplicación en los consultorios de consulta externa. El DQOL permite conocer la satisfacción, impacto y preocupación que tiene el paciente diabético [22]. La aplicación del instrumento fue realizada por las investigadoras a los pacientes que después de informar el propósito de la investigación aceptaron participar.

La información recolectada fue revisada y verificada para identificar el diligenciamiento completo de cada instrumento. Seguidamente se diseñó y alimentó la base de datos en Excel. El paquete estadístico utilizado para el análisis de la muestra fue PASW statistics versión 18 (SPSSS). Este análisis estuvo basado en medidas de frecuencia para variables nominales y ordinales, y medidas de tendencia central y dispersión para el caso de variables cuantitativas [22].

Para el análisis de la consistencia interna del instrumento por subescalas se realizó mediante el cálculo de coeficiente Alpha de Cronbach, utilizando el SPSS18 de la siguiente manera: 1. Se accedió a: Analizar > Escala > Análisis de Fiabilidad 2. Se seleccionaron las variables a analizar aceptándolas para luego obtener el valor del Alfa de Cronbach. Posteriormente se procedió a calcular el análisis factorial confirmatorio por extracción de los de componentes principales con rotación Varimax mediante la utilización del paquete estadístico SPSS18 de cada variable de la siguiente forma: 
1. Analizar/Reducción de Datos > Análisis Factorial 2. >Seleccionar> Descriptivos> Estadísticos> Solución inicial 3. >Matriz de correlaciones $>$ Coeficientes; Niveles de significación $>$ KMO y prueba de esfericidad de Bartlett. 4. > Extracción $>$ Método> Componentes principales $>$ Analizar $>$ Matriz de correlaciones. 5.> Extraer> Mostrar> Solución factorial sin rotar; Gráfico de sedimentación 6. >- Rotación> seleccionar los métodos de rotación 7.> Mostrar (Visualización en SPSS18): Solución rotada eligiendo el método de rotación varimax.

\section{Resultados y análisis}

\subsection{Características sociodemográficas}

La muestra estuvo integrada por 287 pacientes con Diabetes Mellitus Tipo 2, el rango de edad se encuentra entre los 50 y 80 años y más; los grupo etáreos de mayor representatividad es el de 50-54 años (23.3 \%) y 70-74 años (23.0\%); La edad promedio en el grupo de pacientes es de 65.9 años con un coeficiente de variación del 10.1 años. El $42.5 \%$ de los encuestados se encuentran en una edad inferior o igual a 64 años. La muestra estuvo representada en un $54.7 \%$ por el género femenino, y en un 45.3 $\%$ por el género masculino.

El $59.9 \%$ de los pacientes viven con pareja actualmente (casados o en unión libre), mientras que el $40.1 \%$ no tiene pareja en la actualidad (solteros, divorciados y/o viudos). Más de la mitad de los pacientes cuentan con un vínculo afectivo estable situación a favor para estos individuos por contar con un apoyo familiar cercano en relación con el tratamiento a seguir para mantener un estado de salud óptimo.

La Escolaridad promedio del grupo de pacientes diabéticos que participaron en el estudio es de 4.64 con un coeficiente de variación de 3.3 años, el $30 \%$ tiene estudios de primaria incompleta y el $51.6 \%$ realizaron estudios de primaria. El $50 \%$ de los pacientes lleva 9 años o menos con la enfermedad [22].

\subsection{Consistencia interna del instrumento DQOL}

Establecer la consistencia interna de una escala es una aproximación a la validación del constructo y consiste en la cuantificación de la correlación que existe entre los ítems que la componen [7]. La consistencia interna de los instrumentos se puede analizar de manera indirecta, mediante la revisión de la relación que existe entre los ítems que componen una escala; es decir, si cada ítems que hace parte de una escala presenta una excelente consistencia interna o interrelación entre sí se puede concluir que la escala tiene un constructo válido; si, por el contrario, se encuentra que los ítems se correlacionan de manera muy pobre, entonces se pueden interpretar los resultados de tres formas: la primera, la escala no mide el constructo que quiere medir; la segunda, la conceptualización teórica en que está basado el constructo es incorrecta, y la tercera, que el diseño experimental era inadecuado y falló en probar la hipótesis misma [7].

No existe un estándar para medir la calidad de vida, se han diseñado algunas escalas que presenta una buena consistencia interna en las distintas poblaciones donde se ha usado, con un valor del Alfa de Cronbach adecuado, o sea, los ítems que la componen guardan una buena correlación entre ellos indicando que tienen un constructo válido. Este método evalúa el error factorial específico y el error por respuesta al azar, los cuales están distribuidos independientemente dentro de los ítems y tienden a cancelarse mutuamente cuando los puntajes de los ítems son sumados La escala es aplicada a los sujetos en un momento puntual y mide, en pocas palabras, la interrelación de los ítems de la escala, para lo cual la fórmula más usada es el coeficiente alfa de Cronbach [10].

La versión en español del DQOL no ha sido validada en el contexto del país, siendo este
Enero - Junio 2015 ISSN 0122-820X PP: 51-66 
Consistencia interna de la versión en español del cuestionario medida de calidad de vida de la diabetes, en Cúcuta (Colombia)

trabajo la primera versión que valida el instrumento medida de calidad de vida específica para Diabetes DQOL. El instrumento muestra una consistencia interna adecuada indicando que los ítems del instrumento son homogéneos y las respuestas son coherentes y relacionadas, midiendo todas las variables de calidad de vida en pacientes con patología de diabetes. La fiabilidad que tiene para la población colombiana específicamente del Norte de Santander es alta mostrando que todos los ítems permiten valorar de manera precisa la calidad [23].

Tabla I. Relación de las puntuaciones promedio obtenidas en cada una de las subescalas del Cuestionario medida de calidad de vida específica para Diabetes (DQOL). Norte de Santander (Colombia)

\begin{tabular}{|c|c|c|c|c|c|c|}
\hline \multirow{2}{*}{ SUB-ESCALA } & \multirow{2}{*}{ ITEMS } & \multirow{2}{*}{$\begin{array}{l}\text { POSIBLE PUNTUACIÓN } \\
\text { MÁXIMA }\end{array}$} & \multicolumn{2}{|c|}{ PUNTUACION BRUTA } & \multicolumn{2}{|c|}{$\begin{array}{l}\text { PUNTUACION } \\
\text { CORREGIDA }\end{array}$} \\
\hline & & & Promedio & $\begin{array}{l}\text { Desviación } \\
\text { Típica }\end{array}$ & Promedio & $\begin{array}{l}\text { Desviación } \\
\text { Típica }\end{array}$ \\
\hline SATISFACCIÓN CON EL TRATAMIENTO & 15 & 75 & 33.7 & 8.2 & 56.3 & 8.2 \\
\hline IMPACTO EN EL TRATAMIENTO & 20 & 100 & 41.1 & 7.5 & 78.9 & 7.5 \\
\hline $\begin{array}{l}\text { PREOCUPACIÓN POR ASPECTOS SOCIALES Y } \\
\text { VOCACIONALES }\end{array}$ & 7 & 35 & 10.6 & 3.2 & 31.4 & 3.2 \\
\hline $\begin{array}{l}\text { PREOCUPACIÓN ACERCA DE LOS EFECTOS } \\
\text { FUTUROS DE LA ENFERMEDAD }\end{array}$ & 4 & 20 & 9.9 & 2.1 & 14.1 & 2.1 \\
\hline CALIDAD DE VIDA (TOTAL DQOL) & 46 & 230 & 95.3 & 16.0 & 180.7 & 16.0 \\
\hline
\end{tabular}

Fuente: [19]

En la Tabla I se presentan resultados con las dos formas de calificación del instrumento, la puntuación directa que corresponde al cálculo con la forma de calificación original, en donde un mayor puntaje significa deterioro de calidad de vida, y la puntuación corregida corresponde a la transformación de la puntuación bruta de cada subescala en una escala de 100 puntos, de modo que 100 representa la mejor calidad de vida y cero significa mayor deterioro de este constructo [24]. El dendograma teniendo en cuenta la correlación entre variables, muestra la asociación existente entre los indicadores. Se observan cuatro cluster, validando la organización de las mismas. De igual manera correlograma muestra coeficientes de correlación que oscilan entre 0,22 y 1 , y correlaciones inter-elementos que oscilan entre 0,54 y 0,99 .

Tabla II. Consistencia interna mediante Alfa de Cronbach del cuestionario medida de calidad de vida específica para Diabetes (DQOL).Norte de Santander (Colombia)

\begin{tabular}{|l|c|c|}
\hline \multicolumn{1}{|c|}{ ESCALA } & ITEMS & $\begin{array}{c}\text { ALPHA } \\
\text { CRONBACH }\end{array}$ \\
\hline SATISFACCION CON EL TRATAMIENTO & 15 & 0.83 \\
\hline IMPACTO EN EL TRATAMIENTO & 20 & 0.78 \\
\hline PREOCUPACION POR ASPECTOS SOCIALES Y VOCACIONALES & 7 & 0.72 \\
\hline PREOCUPACION ACERCA DE LOS EFECTOS FUTUROS DE LA ENFERMEDAD & 4 & 0.29 \\
\hline CALIDAD DE VIDA (TOTAL DQOL) & 46 & 0.88 \\
\hline
\end{tabular}

Fuente: [19]

El Coeficiente Alfa de Cronbach, fue descrito en 1951 por Cronbach Lee C, se puede concebir como la medida en la cual algún constructo, concepto o factor medido está presente en cada ítem, o como el índice usado para medir la confiabilidad del tipo consistencia interna de una escala, es decir, para evaluar la magnitud en que los ítems de un instrumento están correlacionados [7]. Este coeficiente requiere una sola administración del instrumento de medición y produce valores que oscilan entre $\mathrm{O}$ y 1 , cuanto más cerca se encuentre el valor del alfa a 1 mayor es la consistencia interna de los ítems analizados [11]. Su ventaja reside en que no es necesario dividir en dos mitades a los ítems del instrumento de medición, simplemente se aplica la medición y se calcula el coeficiente, Este índice de consistencia interna puede ser calculado manualmente o en Excel de dos formas: 1) Mediante la varianza de los ítems o 2) Mediante la matriz de correlación [9]. 
Dado que los atributos de confiabilidad tienen que ver con el grado de consistencia entre los resultados que son obtenidos en dos tiempos independientes de prueba, están expresados a menudo en términos de coeficientes de correlación. El coeficiente de confiabilidad expresa la relación entre la varianza de error, la varianza verdadera y el resultado observado, una correlación 0 indica que no hay relación, cuando la varianza de error de un instrumento de medición es alta, el coeficiente de confiabilidad va a ser cercano a 1 , esto indica que el instrumento es más confiable. Por ejemplo; un coeficiente de 0.79 quiere decir que la varianza de error es pequeña y que la herramienta tiene poca medida de error, por otro lado si el coeficiente de confiabilidad es 0.49 el error de la varianza es alto y la herramienta tiene un problema de error de medida. Para que una herramienta sea considerada confiable un nivel de 0.70 cuando el instrumento esta recién desarrollado o en caso contrario 0.80 son aceptados como un nivel de confiabilidad aceptable [25].

El valor mínimo aceptable para el coeficiente Alfa de Cronbach es 0.7; por debajo de ese valor la consistencia interna de la escala utilizada es baja. Este valor manifiesta la consistencia interna, es decir, muestra la correlación entre cada una de las preguntas; un valor superior a 0.7 revela una fuerte relación entre las preguntas, un valor inferior revela una débil relación entre ellas. No es común, pero el Alfa de Cronbach puede arrojar un valor negativo, esto indica un error en el cálculo o una inconsistencia de la escala [11].

Los coeficientes de consistencia interna de Alpha de Cronbach para las diferentes subescalas y para la puntuación total del DQOL en el Departamento Norte de Santander (Colombia) como estudio confirmatorio, oscilaron entre 0,29 a 0,88 ; las escalas de satisfacción con el tratamiento, impacto en el tratamiento, preocupación por aspectos sociales y vocacionales obtienen un valor entre 0.72 y 0.88 como se puede observar en la Tabla II; indicando que tienen un consistencia entre aceptable y buena según lo han determinado autores que han realizado otras interpretaciones de este coeficiente [26], que establecen una escala cuantitativa y cualitativa de los valores del coeficientes de alfa de Cronbach de la siguiente manera Coeficiente alfa $>.9$ es excelente, Coeficiente alfa $>8$ es bueno, Coeficiente alfa $>7$ es aceptable, Coeficiente alfa $>.6$ es cuestionable, Coeficiente alfa $>5$ es pobre y Coeficiente alfa $<5$ es inaceptable [26] . Así mismo otros autores [27], consideran que el valor de fiabilidad en una investigación exploratoria debe ser igual o mayor a 0.6 , y en estudios confirmatorios debe estar entre $0.7 \mathrm{y}$ 0.8 [25], [28].

La escala preocupación acerca de los efectos futuros de la enfermedad obtuvo un valor de 0,29 lo cual indica una interpretación de inaceptable [26]. El carácter crónico y generalmente progresivo de la diabetes, la vuelven una enfermedad muy demandante; ya que para lograr un adecuado control, la persona debe realizar cambios sostenidos en su estilo de vida y esto no siempre es fácil, podrían generarse resistencias. "El curso y desenlace de la enfermedad crónica está influenciado por variables sociales y emocionales, variables que podrán tener un efecto decisivo en la manera en que la enfermedad se desarrolla. Cada fase del padecimiento, enfrenta a la persona con la necesidad de cumplir tareas especiales y se requerirá de diferentes estrategias para su adecuado enfrentamiento y asimilación" [29]. 
Con la intención de analizar la validez del constructo existente, se realizó un análisis factorial confirmatorio con el método de componentes principales y rotación Varimax. Este análisis identificó la correspondiente estructura factorial, corroborando la multidimensionalidad del cuestionario que se compone de 4 factores según la propuesta realizada por sus autores. Para los cuatro factores analizados, se observa en la Tabla III, que el mayor índice de correlación de casi la totalidad de las preguntas corresponde con el factor para el que fueron diseñadas, excepto los ítems b4 y b6 que muestran mayor correlación con el factor satisfacción con el tratamiento, y los ítems d1 y d3 que muestran mayor correlación con el factor impacto en el tratamiento.

El análisis factorial es una técnica que analiza las correlaciones lineales entre las variables. $\mathrm{Si}$ las variables no estuvieran asociadas linealmente, las correlaciones entre ellas serían nulas, no existiendo asociación [14], los valores menores a 0,3 se consideran no significativos [16], en la Tabla III se puede observar que los ítems a1, a6, b6 y b18 obtuvieron correlaciones lineales con valores entre 0.10 y 0.27 lo cual se considera no significativo, es decir la relación es nula con el factor donde se encuentran establecidos. Así mismo los ítems a2,a3,a4,a7,a8,a9, a10,a11,a1 2,a13,a14,a15,b1,b2,b7,b10,b11,b12,b13,b17 ,b19,b20,c1,c2,c4,c5,c6,c7,d1 y d3 mostraron correlaciones lineales con valores entre $0.5 \mathrm{y}$ 0.7 lo cual indica un aporte significativo. Se destaca los valores obtenidos en los ítems d2 y d 4 , los cuales fueron superiores a 0.8 siendo considerado un resultado relevante.

De otra parte en el análisis factorial realizado al DQOL se encontró que los ítems b6 y b8 revelaron correlaciones de $(-0.54)$ y $(-0.06)$ respectivamente, indicando un peso negativo para el factor 2 (Impacto en el tratamiento) seguramente fundamentado en que los ítems abordan aspectos muy personales y no el manejo integral que debe brindar la institución de salud donde debe incluir educación al paciente y su familia mediante la orientación en forma sencilla respecto a lo que es la enfermedad, las características de la alimentación y el ejercicio que debe realizarse; y el seguimiento institucional sustentado en la vigilancia de las condiciones clínicas y metabólicas, seguimiento a complicaciones y la prescripción apropiada de los medicamentos [30]. La matriz de factores contiene las ponderaciones (cargas o pesos) de cada variable. Estos pesos corresponden a las correlaciones de cada una de las variables sobre cada factor. Los valores se obtienen en términos absolutos (debido a que la correlación puede ser positiva o negativa) indicando la representatividad de la variable sobre el factor; por lo tanto, va valores altos representan mayores contribuciones, y valores bajos muestran baja representatividad [16].

Según el estudio realizado la diabetes tipo 2 , es una patología que tiene su inicio en personas relativamente jóvenes en etapa productiva situación que hace evidente de cómo los factores de riesgo propios de los estilos de vida están llevando a la presencia de manifestaciones de esta enfermedad en adultos jóvenes y sobre todo en mujeres quienes epidemiológicamente se ven más afectadas por esta situación de salud. Se encontró que el predominio del género femenino hace a estas personas más propensas a comprometerse en acciones de promoción de la salud [31].

La mayoría de las subescalas presenta una consistencia interna aceptable, excepto la relacionada con preocupación por aspectos futuros de la enfermedad $(\alpha=0,29)$. De forma general se concluye que el instrumento expresa una confiabilidad elevada $(\alpha=$ 0,88 ); esta confiabilidad es semejante con otros estudios obteniendo los coeficientes de consistencia interna del instrumento en rangos de 0.68 a 0.86 [19], y también se encontró una adecuada consistencia interna tanto en las subescalas como en el total del DQOL con un valor de 0.89 [6].
Enero - Junio 2015 ISSN 0122-820X PP: 51-66 
No. 1

Enero - Junio 2015

ISSN 0122-820X

PP: 51-66
Según el instrumento DQOL la subescala más afectada es la del impacto en el tratamiento con un promedio de 41.1 y la menos afectada es la preocupación por los efectos futuros de la enfermedad, este resultado difiere con otros estudios encontrando que la subescala más afectada fue satisfacción con el tratamiento, y la menos afectada fue impacto de la diabetes [32]; en el área psicosocial, el 60 $\%$ de los sujetos no mostró deterioro y en $15 \%$ fue severo, $\mathrm{p}<0.003$ [12]; también se encontró que la subescala más afectada es la relacionada con el control de la diabetes y ansiedad-preocupación [33], mientras que el aspecto que ha obtenido la valoración más desfavorable es la satisfacción con el tratamiento [22].

Se encontraron diferencias significativas en la dimensión de calidad de vida "Satisfacción con el Tratamiento" en los pacientes con respecto al estado civil; los pacientes sin pareja obtuvieron una mayor puntuación promedio que aquellos pacientes con pareja ( $35.4 \pm 7.4$ vs. $32.6 \pm 8.5 ; p=0.001)$, lo cual significa un mayor deterioro en la calidad de vida con respecto a esta subescala, en personas solteras, divorciadas o separadas. A nivel general, existen diferencias significativas en la calidad de vida de los pacientes con respecto al estado civil, observándose mayor puntuación promedio en pacientes sin pareja, que en aquellos con una relación estable (97.8 \pm 14.3 vs. $93.6 \pm 17 ; p=0.03)$ [22].

Se señala que la "enfermedad en el ser humano además del componente patológico consta de uno ecológico en el que la familia y los amigos constituyen el contexto social más significativo y duradero. Un individuo con diabetes se encuentra todos los días ante sus propias necesidades, dirigiéndose casi siempre a su familia o personas cercanas en busca de ayuda y consejo para solucionar sus problemas de salud; la respuesta que le brinden influirá positiva o negativamente sobre su conducta terapéutica y, por lo tanto, en el control de la diabetes" [34].
La calidad de vida global en esta investigación es considerada como la sensación de bienestar que expresa el paciente diabético con relación a la salud, tratamiento ordenado, complicaciones y efectos de la enfermedad. La calidad de vida relacionada con la salud es considerada como un proceso dinámico y cambiante que incluye interacciones continúas entre el paciente y su medio ambiente [35]. De acuerdo a este concepto, la calidad de vida en una persona enferma es la resultante de la interacción del tipo de enfermedad y su evolución, la personalidad del paciente, el grado de cambio que inevitablemente se produce en su vida, el soporte social recibido y percibido y la etapa de la vida en que se produce la enfermedad [35]. Los resultados de la investigación muestran que el $62.3 \%$ de los pacientes manifiestan tener una calidad de vida MEDIA el $37.6 \%$ expresa poseer una calidad de vida ALTA y ningún paciente tiene calidad de vida BAJA.

\section{Conclusiones}

El instrumento aplicado DQOL (Diabetes Quality of Life) permitió conocer la validez y confiabilidad del Cuestionario medida de calidad de vida específica para Diabetes (DQOL en población diabética Colombiana (Departamento Norte de Santander), mostrando que es un instrumento válido para conocer la calidad de vida, debido a que esta escala a nivel global tiene una alta fiabilidad y presenta una alta correlación entre cada ítem con el factor que evalúa.

El coeficientes de consistencia interna del instrumento osciló entre 0,29 a 0,88. La mayoría de las subescalas presenta una consistencia interna aceptable, excepto la relacionada con preocupación por aspectos futuros de la enfermedad $(\alpha=0,29)$. A nivel general se concluye que el instrumento expresa una confiabilidad elevada $(\alpha=0,88)$.

\section{Agradecimientos}

A Las directivas de la ESE IMSALUD por 
su colaboración incondicional y autorización para obtener la información requerida en el estudio

A las enfermeras de las Unidades Básicas de Comuneros, Libertad y Puente Barco Leones de la ESE IMSALUD; por su colaboración durante el desarrollo del estudio.

\section{Referencias}

[1] Departamento Administrativo Nacional de Estadísticas (DANE). Boletín De Prensa. Encuesta de calidad de vida 2008. Bogotá: Dirección de Difusión, Mercadeo y Cultura Estadística DANE. 2009. [Online]. Disponible en: http:// www.dane.gov.co/files/investigaciones/ condiciones_vida/calidad_vida/ Boletin_Prensa_ECV_2008.pdf.

[2] S. Galán Cueva, L. S. Alemán de la Torre, M. P. Guevara Gasca, Z. Z. Landeros Perez. "Afectación de la calidad de vida en personas con enfermedades crónicas y degenerativas”. En: Resúmenes. Cuarta Reunión Nacional de Investigación en Psicología. 21 y 22 de junio de 2012. México: Sistema Mexicano de Investigación en Psicología (SMIP). 2012. pp. 1-6. [Online]. Disponible en: http://tagit.idex.mx/smip2010/contenido/resumenes/procesos_psicosociales_clinica_y_salud/Sergio_Galan_ Cuevas_y_cols.pdf.

[3] M. Puig Lobet. "Cuidados y calidad de vida en Vilafranca del Penedès: los mayores de 75 y más años atendidos por el servicio de atención domiciliaria y sus cuidadores familiares". Tesis Doctoral. Barcelona: Universitat de Barcelona. 2009. [Online]. Disponible en: http://diposit.ub.edu/dspace/bitstream/2445/43013/1/MPLL_TESIS. pdf.

[4] J. L. García Gómez. "Evaluación de la calidad de vida en los pacientes con diabetes mellitus tipo 1: el caso del Hospital Universitario nuestra Señora de Candelaria de Tenerife". Tesis Doctoral. Tenerife: Servicio de Publicaciones, Universidad de La Laguna. 2006.

[5] M. Gómez Vela, E. N. Sabeh. "Calidad de vida. Evaluación del concepto y su influencia y la práctica", Integra, vol. 21, no.1, pp. 25-7, Jun 2000. [Online]. Disponible en: http://campus.usal. es/ inico/investigacion/invesinico/calidad.htm.

[6] A. Machado Romero, M. T. Anarte Ortiz, M. S. Ruiz de Adana Navas, "Predictores de calidad de vida en pacientes con diabetes mellitus Tipo 1", Clínica y Salud, vol. 21, no. 1, pp. 35-47, 2010. [Online]. Disponible en: http:// scielo.isciii.es/scielo.php?pid=S1130$52742010000100004 \&$ script $=$ sci_art text.

[7] H. C. Oviedo, A. Campo Arias, "Aproximación al uso del coeficiente alfa de Cronbach", Revista Colombiana de Psiquiatría, vol. 34, no.4, pp.572580, 2005.

[8] D. Frías Navarro. Apuntes de SPSS. Valencia: Universidad de Valencia, 2014. [Online]. Disponible en: http:// www.uv.es/ friasnav/ApuntesSPSS. pdf. $2-820 \mathrm{X}$ P: 51-66

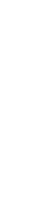


No. 1
[11] J. A. Bojórquez Molina, L. López Aranda, M. E. Hernández Flores, E. Jiménez López. "Utilización del alfa de Cronbach para validar la confiabilidad de un instrumento de medición de satisfacción del estudiante en el uso del software Minitab MISP". En: $11^{\text {th }}$ LACCEI Latin American and Caribbean Conference for Engineering and Technology (LACCEI'2013) "Innovation in Engineering, Technology and Education for Competitiveness and Prosperity" August 14 - 16, 2013 Cancun, Mexico: Latin American and Caribbean Consortium of Engineering Institutions; 2013. p. 1-9.

[12] J. L. De Los Ríos Castillo, J. J. Sánchez Sosa, P. Barrios Santiago, V. Guerrero Sustaita, "Calidad de vida en pacientes con diabetes mellitus tipo 2", Rev Med IMSS, vol. 42, no. 2, pp. 109-116, 2004.

[13] M. L. Garmendia. "Análisis factorial: una aplicación en el cuestionario de salud general de Goldberg, versión de 12 preguntas", Rev Chil Salud Pública, vol. 11, no. 2, pp. 57-65, 2007.

[14] M. J. Rodríguez Jaume, R. Morar Catala. "Capítulo 8. Análisis Factorial". En: Estadística informática: casos y ejemplos con el SPSS. M. J. Rodríguez Jaume, R. Morar Catala. Alicante: Universidad De Alicante, Servicio de $\mathrm{Pu}-$ blicaciones: 2002. p. 126-142.

[15] J. A. Pérez Gil, S. Chacón Moscoso, R. Moreno Rodríguez, "Validez de constructo: el uso de análisis factorial exploratorio-confirmatorio para obtener evidencias de validez", Psicothema, vol. 12, Supl. 2, pp. 442-446, 2000.

[16] C. Méndez Martínez, M. A. Rondón Sepúlveda, "Introducción al análisis factorial exploratorio", Rev. Colomb. Psi- quiat, vol.41, no.1, pp. 197-207, 2012.

[17] J. M. López Carmona, R. Rodríguez Moctezuma, "Adaptación y validación del instrumento de calidad de vida Diabetes 39 en pacientes mexicanos con diabetes mellitus tipo 2", Salud Pública de México, vol. 48, no. 3, pp. 200-211, 2006.

[18] E. Velarde Jurado, C. Avila Figueroa, "Evaluación de la calidad de vida", Salud Pública de México, vol. 44, no. 4, pp. 349-361. 2002. Disponible en: http://www.scielo.org.mx/scielo. php?script $=$ sci_arttext\&pid=S003636342002000400009\&lng=es.

[19] R. Robles García, J. Cortázar, J. J. Sánchez Sosa, F. Páez Agraz, H. Nicolini Sánchez, "Evaluación de la calidad de vida en Diabetes Mellitus Tipo 2: propiedades psicométricas de la versión en español del DQOL", Psicothema, vol. 15, no. 2, pp. 247-252, 2003.

[20] The DCCTResearch Group, "Reliability and validity of a diabetes quality-of-life measure for the Diabetes Control and Complications Trial (DCCT)", Diabetes Care, vol. 11, pp. 725-732, 1988.

[21] M. Millan Guasch. "Validación de un cuestionario de calidad de vida del paciente diabético y material didáctico para la educación en diabetes: dos instrumentos complementarios". Tesis Doctoral. Barcelona: Universidad de Barcelona. 1996.

[22] M. del C. Gervas Alcalaya. "Calidad de vida y automanejo de la enfermedad en diabéticos insulinodependientes de Valladolid". Tesis Doctoral. Valladolid: Universidad de Valladolid; 1997. [Online]. Disponible en: http://www. 
cibernetia.com/tesis es/CIENCIAS MEDICAS/MEDICINA INTERNA/ ENDOCRINOLOGIA/5.

[23] F.J. Abad, J. Garrido, J. Olea, V. Ponsoda. Introducción a la psicometría: Teoría Clásica de los Test y Teoría de la Respuesta al Ítem. Madrid: Universidad Autónoma de Madrid, 2006. [Online]. Disponible en: https://www.uam. es/personal_pdi/psicologia/cadalso/ Docencia/Psicometria/Apuntes/ tema1TyP_4.pdf.

[24] M. T. Oviedo Gómez, L. M. Reidl Martínez, "Predictores psicológicos individuales de la calidad de vida en diabetes tipo 2", Revista Mexicana de Psicología, vol. 24, no. 1, pp. 31-42, 2007. [Online]. Disponible en: http://www.redalyc. org/articulo.oa? $\mathrm{id}=243020635005$.

[25] D. Iacobucci, A. Duhachek, “Advancing Alpha: Measuring Reliability With Confidence", Journal of Consumer Psychology, vol. 13, no. 4, pp. 478-487, 2003.

[26] D. George, P. Mallery. SPSS for Windows step by step: A Simple Guide and Reference. 11.0 Update. $4^{\mathrm{a}}$ ed. Boston: Allyn \& Bacon, 2003.

[27] J. Huh, D. E. Delorme, L. N. Reid, "Perceived third-person effects and consumer attitudes on prevent ing and banning DTC advertising", Journal of Consumer Affairs, vol. 40, no. 1, pp. 90-104, 2006.

[28] N. Schmitt. "Uses and Abuses of Coefficient Alpha", Psychological Assessment, vol. 8, no. 4, pp. 350-353, 1996.

[29] S. Vinaccia, L. M. Orozco, "Aspectos psicosociales asociados con la calidad de vida de las personas con enfermedades crónicas", Diversitas, vol. 1, no. 2, pp. 125-137, 2005.

[30] M. A. Oviedo Mota, F. Espinosa Larrañaga, H. Reyes Morales, J. A. Trejo y Pérez, E. Gil Velázquez. Enfermedades crónicas. Guía clínica para el diagnóstico y el manejo de la diabetes mellitus tipo 2. [Online]. Disponible en http://www.sld.cu/ galerias/pdf/sitios/diabetes/diabmellt2. pdf

[31] J. D. Gualdrón Flórez. Factores básicos condicionantes del autocuidado asociados a la capacidad potencial para auto cuidarse y promover la salud en personas de 18 años y más que viven en situación de enfermedad cardiovascular y asistieron a la consulta externa de cardiología del Hospital Nacional Rosales. En: I Foro Regional de Investigación; El Salvador, 2003.

[32] V. Cárdenas, C. Pedraza, R. Lerma, "Calidad de vida del paciente con diabetes mellitus tipo2". Ciencia UANL. Julio-septiembre, 2005; vol. 8, no. 3, pp. 351-357.

[33] M. González Suarez, V. Rivas Acuña. Calidad de vida de los adultos con diabetes mellitus tipo 2. Semana de divulgación y video científico 2008, pp. 392-397 Universidad Juárez Autónoma de Tabasco, México. 2008.

[34] I. de la A. Valadez Figueroa, M. G. Alderete Rodríguez, N. Alfaro Alfaro, "Influencia de la familia en el control metabólico del paciente diabético tipo II", Salud Pública México, vol. 35, no. 5, pp. 464-470, 1993.

[35] L. Schwartzmann, "Calidad de vida
Enero - Junio 2015 ISSN 0122-820X PP: 51-66 
Enero - Junio 2015 ISSN 0122-820X

PP: 51-66

relacionada con la salud: aspectos conceptuales", Ciencia y Enfermería. 2003, vol. 9, no. 2, pp. 09-21. [Online]. Disponible en: http://www.scielo.cl/scielo. php?script $=$ sci_arttext\&pid $=\mathrm{S} 0717$ $5532003000200002 \& \operatorname{lng}=\mathrm{es} \& n r m=$ iso. 